\title{
Age-related changes in photosensitive melanopsin-expressing retinal ganglion cells correlate with circadian rhythm impairments in sighted and blind rats
}

\author{
Pedro Lax ${ }^{a}$, Gema Esquiva ${ }^{a}$, Lorena Fuentes-Broto ${ }^{\mathrm{b}, \mathrm{c}}$, Francisco Segurac,d, Ana Sánchez-Cano ${ }^{\mathrm{e}}$, Nicolás Cuenca ${ }^{\mathrm{a}, \mathrm{f}}$, \\ and Isabel Pinillac,g \\ aDepartment of Physiology, Genetics and Microbiology, University of Alicante, Alicante, Spain; ${ }^{b}$ Department of Physiology, University of \\ 5 Zaragoza, Zaragoza, Spain; 'Aragon Institute for Health Research (IIS Aragón), Zaragoza, Spain; 'Department of Surgery, University of \\ Zaragoza, Zaragoza, Spain; eDepartment of Applied Physics, University of Zaragoza, Zaragoza, Spain; fInstitute Ramón Margalef, University of \\ Alicante, Alicante, Spain, and; 9Department of Ophthalmology, Lozano Blesa University Hospital, Zaragoza, Spain
}

\section{ABSTRACT}

The melanopsin system consists of intrinsically photosensitive retinal ganglion cells containing the photopigment melanopsin (mRGCs). These mRGCs mediate several non-image-forming visual functions, including light entrainment of circadian rhythms. Here we evaluate age-related alterations of the melanopsin system and circadian rhythms in $\mathrm{P} 23 \mathrm{H}$ line $1(\mathrm{P} 23 \mathrm{H}-1)$ rats, a rodent model of retinitis pigmentosa (RP). In homozygous $\mathrm{P} 23 \mathrm{H}-1$ rats and wild-type control rats from the same genetic background (Sprague-Dawley), body temperature and locomotor activity were continuously monitored at 10-min intervals for 7 days, once every 4-5 weeks, between 2 and 24 months of age, using a telemetry transmitter. The distribution and number of mRGCs were assessed in control rats at 12,18 , and 24 months of age and in $\mathrm{P} 23 \mathrm{H}-1$ rats aged $12,18,24$, and 30 months by immunostaining whole-mount retinas with antibodies against melanopsin. The mean density of mRGCs in control rats showed no significant variations when evaluated at 12 and 18 months of age, and fell by approximately $56 \%$ between 18 and 24 months of age. Meanwhile, a significant decrease in the mean number of mRGCs was found in 18-month-old $\mathrm{P} 23 \mathrm{H}-1$ rats as compared to 18 -month-old control rats ( $81 \%$ decrease). Parametric and non-parametric analyses of the records showed a gradual age-dependent weakening of body temperature and locomotor activity circadian rhythms robustness in both control and $\mathrm{P} 23 \mathrm{H}-1$ rats from 2 to 24 months of age. However, body temperature and locomotor activity circadian patterns were less robust throughout the experiment in $\mathrm{P} 23 \mathrm{H}-1$ as compared to control rats, with lower amplitude, weaker coupling strength to environmental zeitgebers and higher fragmentation of the rhythms. The present study shows that the degeneration of photoreceptors and inner retinal neurons, characteristic of $\mathrm{RP}$, has age-related degenerative effects on the melanopsin system and is associated with weaker circadian patterns.

\section{Introduction}

The melanopsin system consists of retinal ganglion cells containing the photopigment melanopsin (Provencio et al., 2000). This photosensory brightness-sensing system mediates several nonimage-forming visual functions, including light entrainment of circadian rhythms and pupillary responses to light (Lucas et al., 2001; Panda et al., 2003). In mammals, melanopsin-expressing retinal ganglion cells (mRGCs) are intrinsically photosensitive and provide direct photic information to the suprachiasmatic nuclei (the master circadian clock) through the retinohypothalamic tract (Gooley et al., 2001; Hattar et al., 2002).

\section{KEYWORDS}

Aging; neurodegenerative diseases; $\mathrm{P} 23 \mathrm{H}$ rat; retinitis pigmentosa

CONTACT Pedro Lax pedro.lax@ua.es D Department of Physiology, Genetics and Microbiology, University of Alicante, Alicante, Spain. Phone: +34 965 
60 and blindness in humans are thereby associated with circadian disorders that depend on the degree to which light perception is conserved (de Zavalia et al., 2011; Gonzalez Fleitas et al., 2015; Lahouaoui et al., 2014; Lockley et al., 2007). The higher the degree of light perception a subject has, the more likely their circadian system is to be entrained with normally phased circadian rhythms (Skene \& Arendt, 2007).

Retinitis pigmentosa (RP) is a heterogeneous group of retinal degenerative disorders of a polymorphic hereditary origin that cause a progressive loss of retinal function and represent a major cause of blindness. Approximately 20-25\% of patients with autosomal dominant RP have a mutation in the rhodopsin gene, one of the most common rhodopsin mutations being the $\mathrm{P} 23 \mathrm{H}$, which accounts for approximately one-third of such cases in the USA (Dryja et al., 2000). P23H transgenic albino rats suffer from a progressive rod degeneration initially associated with normal cone function, which is consistent with the clinical findings in $\mathrm{P} 23 \mathrm{H}$ patients (Cuenca et al., 2004; Machida et al., 2000; Pinilla et al., 2005). The loss of photoreceptors is accompanied by degenerative changes in the inner retina (Cuenca et al., 2014; Cuenca et al., 2004; Marc et al., 2003; Puthussery $\&$ Taylor, 2010) and a substantial degeneration of retinal ganglion cells (Garcia-Ayuso et al., 2010; Kolomiets et al., 2010). In previous studies on $\mathrm{P} 23 \mathrm{H}-3$ rats, we demonstrated that retinal degeneration positively correlates with the occurrence of circadian dysfunctions (Lax et al., 2011), and that melanopsin-containing ganglion cells degenerate in advanced stages of the disease (Esquiva et al., 2013; Garcia-Ayuso et al., 2015).

Considering the profound impact of aging on human visual function and ophthalmic diseases (Dagnelie, 2013; Klein \& Klein, 2013), it is important to expand our understanding of the link 100 between pathological changes in the eye and circadian rhythm impairments. Nevertheless, there have been very few longitudinal studies on circadian rhythms in retinal degenerative diseases. The aim of the present research is, therefore, to jointly 105 examine the effects of retinal degeneration and aging on both the melanopsin system and circadian photoentrainment, by simultaneously evaluating mRGC degeneration and circadian rhythms of body temperature and locomotor activity in Sprague-Dawley and $\mathrm{P} 23 \mathrm{H}$ rats. In this study, we used $\mathrm{P} 23 \mathrm{H}$ line $1(\mathrm{P} 23 \mathrm{H}-1)$ transgenic albino rats, because their retinal degeneration is faster than in $\mathrm{P} 23 \mathrm{H}-3$ rats, thus ensuring that a higher degree of retinal degeneration was present at each tested age.

\section{Materials and methods}

\section{Animals}

Homozygous $\mathrm{P} 23 \mathrm{H}-1$ rats $(n=14)$, obtained from Dr. M. LaVail (UCSF), were used as a model of RP. Normal Sprague-Dawley rats (genetic background; $n=9$ ) obtained from Harlan Laboratories (Barcelona, Spain) were used as wild-type controls. All animals were bred in a colony at the University of Zaragoza, Spain, and maintained under controlled humidity (60\%), temperature $\left(23 \pm 1^{\circ} \mathrm{C}\right)$ and photoperiod (LD 12:12) conditions. Light cycle illumination varied from 7 to 30 lux, depending on the front-to-back position within the respective cages. Water and dry feed were made available ad libitum. The average life span of laboratory rats is approximately 3 years (Quinn, 2005). No differences in longevity were found between $\mathrm{P} 23 \mathrm{H}-1$ and Sprague-Dawley rats. All animals were housed, handled and the procedures carried out under the Project License PI 12/14 approved by the Ethic Committee for Animal Experiment from the University of Zaragoza. All procedures were performed in accordance with current regulations for the use of laboratory animals (NIH, ARVO, and European Directive 2010/63/UE), in order to minimize animal suffering and limit the numbers used for the experiments.

\section{Body temperature and locomotor activity recording}

Body temperature and locomotor activity were continuously monitored at 10-min intervals for 7 days, once every 4-5 weeks, from 2 to 24 months of age, using a telemetry transmitter (TA-T20; Data Sciences International, St. Paul, MN, USA). Sterilized transmitters were implanted intraperitoneally under aseptic conditions. No mortality or morbidity was 
observed after the surgery. Data signals were collected using specific Dataquest A.R.T. software (Data Sciences International), which coordinated the detection, collection and analysis of the signals.

Body temperature and locomotor activity records were analyzed for each seven-day experimental period using software specifically designed for chron-

160 obiological analysis (El temps, Diez-Noguera, University of Barcelona). Actograms, mean waveforms, and Sokolove-Bushell periodograms were calculated. Furthermore, a non-parametric analysis of the recordings was performed throughout the experiment for seven-day periods, as previously described (Lax et al., 2012; Lax et al., 2011).

Non-parametric variables were primarily proposed by Witting et al. (1990) to quantify the main characteristics of the rest-activity circadian rhythm. 170 The relative amplitude (RA) of the rhythm represents the ratio of the most active 10-h period (M10) to the least active 5-h period (L5) and is calculated as follows: $(M 10-L 5) /(M 10+L 5)$. A 10-h window and another 5-h window were moved across each day to

175 determine periods of the 10 most active hours and the 5 least active hours, respectively. RA scores theoretically range from 0 to 1 , with higher values indicating a circadian rhythm of higher amplitude. Interdaily phase stability (IS) values are considered 180 proportional to the degree of phase homogeneity during the corresponding experimental period, and can be considered a measure of the phase stability of the rhythm over successive days. The IS was derived by normalizing the 24 -h value from the chi-square periodogram. IS scores range from 0 for Gaussian noise to 1 for perfect stability, with low scores representing poor consistency of activity patterns. The intradaily variability (IV) quantifies the fragmentation of periods of activity from periods of rest within

190 a 24-h period. The IV was calculated by taking the ratio of the mean squared first derivate of the data and the population variance of the data. IV scores range from 0 (when the wave was perfectly sinusoidal) to 2 (Gaussian noise) and are typically below 1 . 195 Higher IV values indicate a more fragmented rhythm and reflect shorter periods of rest and activity rather than one extended active period and one extended rest period. The circadian function index (CFI), described by Ortiz-Tudela et al. (2010), takes

account RA, IS and IV scores. IV values was calculated as the average of these three parameters. Consequently, CFI oscillates between 0 (absence of circadian rhythmicity) and 1 (a robust circadian rhythm).

\section{Retinal histology}

\section{Immunoperoxidase labeling}

Animals were sacrificed in the morning, between 10:00 a.m. and 12:00 p.m., by administering a lethal dose of pentobarbital. After marking the dorsal margin of the limbus with a stitch, eyes were enucleated, fixed in $4 \%(\mathrm{w} / \mathrm{v})$ paraformaldehyde during $1 \mathrm{~h}$ at room temperature (RT), washed in phosphate buffer and sequentially cryoprotected in 15, 20, and $30 \%$ sucrose. The cornea, lens and vitreous body were removed, and the eyecups were processed for whole mounts.

Whole-mount retinas were dissected out from the choroid and put through a freeze-thaw procedure by dipping them in liquid nitrogen-cooled isopentane for a few seconds. After thawing in $30 \%$ sucrose, retinas were washed in phosphate buffer. Endogenous peroxidase activity was suppressed by immersion in 1\% hydrogen peroxide (H1009, Sigma, St. Louis, MO, USA) in phosphate buffer (10 minutes, RT). In order to break aldehyde bonds and enhance the permeability of the tissue, the retinas were incubated first in $2.28 \%$ sodium $\mathrm{m}$-periodate (S1878, Sigma) in phosphate buffer (5 minutes, RT) and then in $0.02 \%$ sodium borohydride (163314, Panreac, Barcelona, Spain) in phosphate buffer (5 minutes, RT). After a blocking step $(10 \%$ normal goat serum in phosphate buffer plus $0.5 \%$ Triton X-100 for $1 \mathrm{~h}$ ), flat-mount retinas were incubated for 2 days at $4^{\circ} \mathrm{C}$ in a 1:2000 dilution of the rabbit anti-melanopsin primary antibody (PA1780, Thermo Fisher Scientific Inc., Rockford, IL, USA), washed four times in phosphate buffer (5 minutes, RT), and then incubated for one day at $4^{\circ} \mathrm{C}$ in biotinylated goat anti-rabbit secondary IgG antibody at 1:100 dilution in phosphate buffer plus $0.5 \%$ Triton X-100. The retinas were washed before transferring them to a solution of avidin-biotin peroxidase complex (PK-6100, Elite ABC kit, Vector Laboratories Ltd, Cambridgeshire, UK) in phosphate buffer containing $0.5 \%$ Triton X-100 for 1 day. Finally, the retinas were washed in phosphate buffer and pre-incubated under agitation in the inverted and normalized between 0 and 1 , and CFI 
dark with 3,38-diaminobenzidine tetrahydrocholoride (DAB, D5637, Sigma; $0.5 \mathrm{mg} / \mathrm{ml}$ in phosphate buffer) for 15 minutes and further incubated in fresh DAB solution, with $0.01 \% \mathrm{H}_{2} \mathrm{O}_{2}$ and $0.025 \%$ ammonium nickel (II) sulfate hexahydrate (A1827, Sigma). The DAB reaction was stopped by washing 255 with distilled water (Cuenca \& Kolb, 1998). Whole retinas were flat mounted in Citifluor (Citifluor Ltd), with the ganglion layer side up, and coverslipped for optical microscopy viewing on a Leica DMR microscope (Leica Microsystems).

260 In order to determine the spatial distribution and total number of mRGCs stained in each retina examined, each and every one of the cell bodies labeled with immunoperoxidase in each entire retina were manually traced. To reconstruct the

265 soma and dendritic profiles of individual mRGCs, we manually traced the outlines of the cell body and the dendritic field of representative labeled cell. The images created were subsequently digitalized, using the image-editing software Photoshop 10.0 (Adobe Systems Inc).

\section{Statistical analysis}

Normal distribution and homogeneity of variance were found for the categories of mRGC density; thus, a two-way analysis of variance (ANOVA) 275 was performed to evaluate the effects of genotype (Sprague-Dawley vs. P23H-1) and age (12, 18, 24 and 30 months), as well as the interactions among them. When a 0.05 level of significance was found, post-hoc pairwise comparisons using Bonferroni's test were performed.

For the analysis of age-related changes in circadian values, according to results of the Kolmogorov-Smirnov test, a repeated measures ANOVA was conducted to compare each parameter between the $\mathrm{P} 23 \mathrm{H}-1$ and control group at different ages (2-24 months). The within-subject factor was each circadian parameter at different ages; the between-subject factor was the genotype (Sprague-Dawley vs. P23H-1). Box's test of equality 290 of covariance matrices was verified before interpretation of results. When a violation of the sphericity assumption was observed (Mauchly's test), results were reported using the Greenhouse-Geisser epsilon. Significant differences were further analyzed using Bonferroni post-hoc comparisons. In the correlational analyses between mRGC density and circadian values, Pearson's product moment correlation coefficients were calculated.

Data are reported as the mean \pm standard error of the mean. Values of $P<0.05$ were considered to be statistically significant. All statistical analyses were performed using SPSS 20.0 software (Statistical Package for Social Sciences, Chicago, IL, USA).

\section{Results}

Melanopsin-containing retinal ganglion cells in control and $\mathrm{P} 23 \mathrm{H}-1$ rat retinas

To examine melanopsin expression in both control and $\mathrm{P} 23 \mathrm{H}-1$ rat retinas, whole-mount rat retinas were immunostained with primary polyclonal antibody against melanopsin. mRGCs were distributed throughout the entire retina in control (Figure 1A-C) and P23H-1 (Figure 2A-C) rats, even though a somewhat higher density was observed in the upper-temporal part of the retina. Melanopsin immunoreactivity was present at comparable intensity on the soma, axon, and dendrites of a subpopulation of RGCs on both control (Figure 1D-I) and P23H-1 (Figure 2D-I) rat retinas. Immunofluorescent cell bodies appeared in the ganglion cell layer (GCL) and, to a lesser extent, within the innermost cell row of the inner nuclear layer (INL). Two plexuses of immunofluorescent dendrites were evident within the inner plexiform layer: one occupying the outer margin (outer part of the OFF-sublamina, stratum S1) and the other occupying the inner side (inner part of the ON-sublamina, stratum S5).

\section{Age-related changes in $\mathrm{mRGC}$ density in control and $\mathrm{P} 23 \mathrm{H}-1$ rats}

At 12 months of age, the mean number of melanopsin-containing ganglion cells measured in control Sprague-Dawley rats was $32.9 \pm 3.6 \mathrm{cells} / \mathrm{mm}^{2}$ (2222 \pm 194 cells/retina, $n=7$; Figure 3$)$. This density value showed no significant variation when evaluated at 18 months of age $(37.9 \pm 2.4$ cells $/ \mathrm{mm}^{2}, 2505 \pm 156$ cells/retina, $n=7$; Figure 3 ). However, the mean number of mRGCs in 24month-old control rats $\left(18.8 \pm 1.4 \mathrm{cells} / \mathrm{mm}^{2}\right.$, $1248 \pm 93$ cells/retina, $n=4$; Figure 3 ) was smaller 

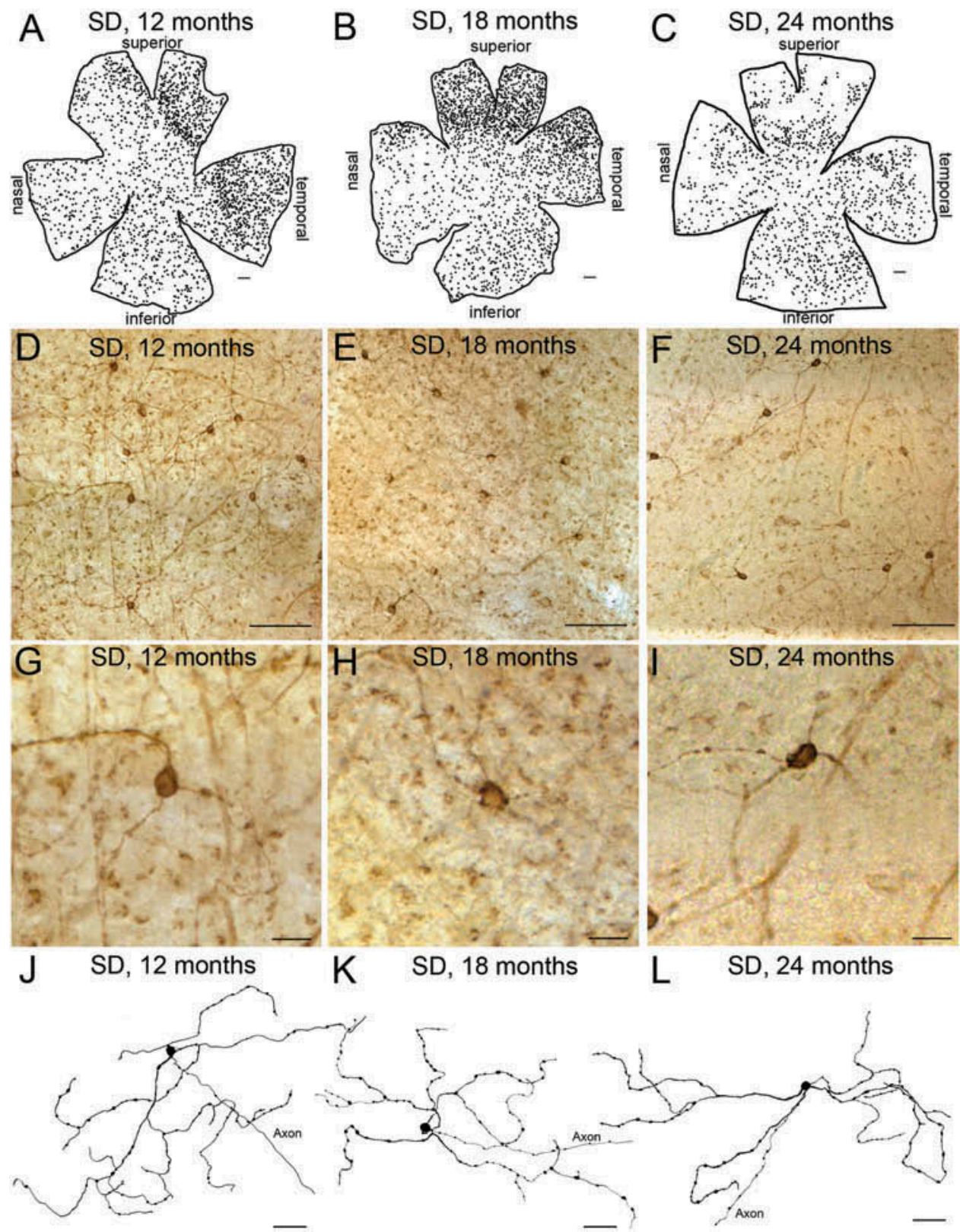

Figure 1. Distribution, morphology and arborization of $m R G C s$ in control Sprague-Dawley rat retinas. (A-C) Representative drawings of whole-mount retinas from Sprague-Dawley (SD) rats at 12 (A), 18 (B), and 24 (C) months of age. Retinas were drawn by hand, showing the location of individual mRGCs labeled with immunoperoxidase. (D-F) Examples of mRGCs in whole-mount rat retinas from Sprague-Dawley rats at 12 (D), 18 (E) and 24 (F) months of age. Each image is the projection of 5 to 10 focal planes between the GCL and the stratum S1 of the IPL. (G-I) High magnification of D-F, respectively. (J-L) Representative drawings of the soma and complete dendritic field of mRGCs from a region of the central retina (between the superior and nasal quadrants) of SpragueDawley rats at $12(\mathrm{~J}), 18(\mathrm{~K})$ and 24 (L) months of age. Scale bars: A-C, $500 \mu \mathrm{m} ; \mathrm{D}-\mathrm{F}, 100 \mu \mathrm{m} ; \mathrm{G}-\mathrm{I}, 20 \mu \mathrm{m} ; \mathrm{J}-\mathrm{L}, 50 \mu \mathrm{m}$.

than that measured in 12- and 18-month-old control rats (ANOVA, Bonferroni's test, $P<0.01$ and $P<0.001$, respectively).

In $\mathrm{P} 23 \mathrm{H}-1$ rat retinas, the mean density of mRGCs found at 12 months of age $(26.3 \pm 4.6$ cells $/ \mathrm{mm}^{2}, 986 \pm 266$ cells/retina, $n=3$; Figure 3 ) was similar to that of control rats of the same age.
However, a significant decrease in the mean number of mRGCs was found in 18-month-old P23H-1 rats $\left(7.3 \pm 0.5\right.$ cells $/ \mathrm{mm}^{2}, 353 \pm 15$ cells/retina, $n=$ 6; Figure 3) as compared to 12- and 18-month-old in both cases). At 24 months of age, the mean number of mRGCs in $\mathrm{P} 23 \mathrm{H}-1$ rats $(8.7 \pm 1.2$ 

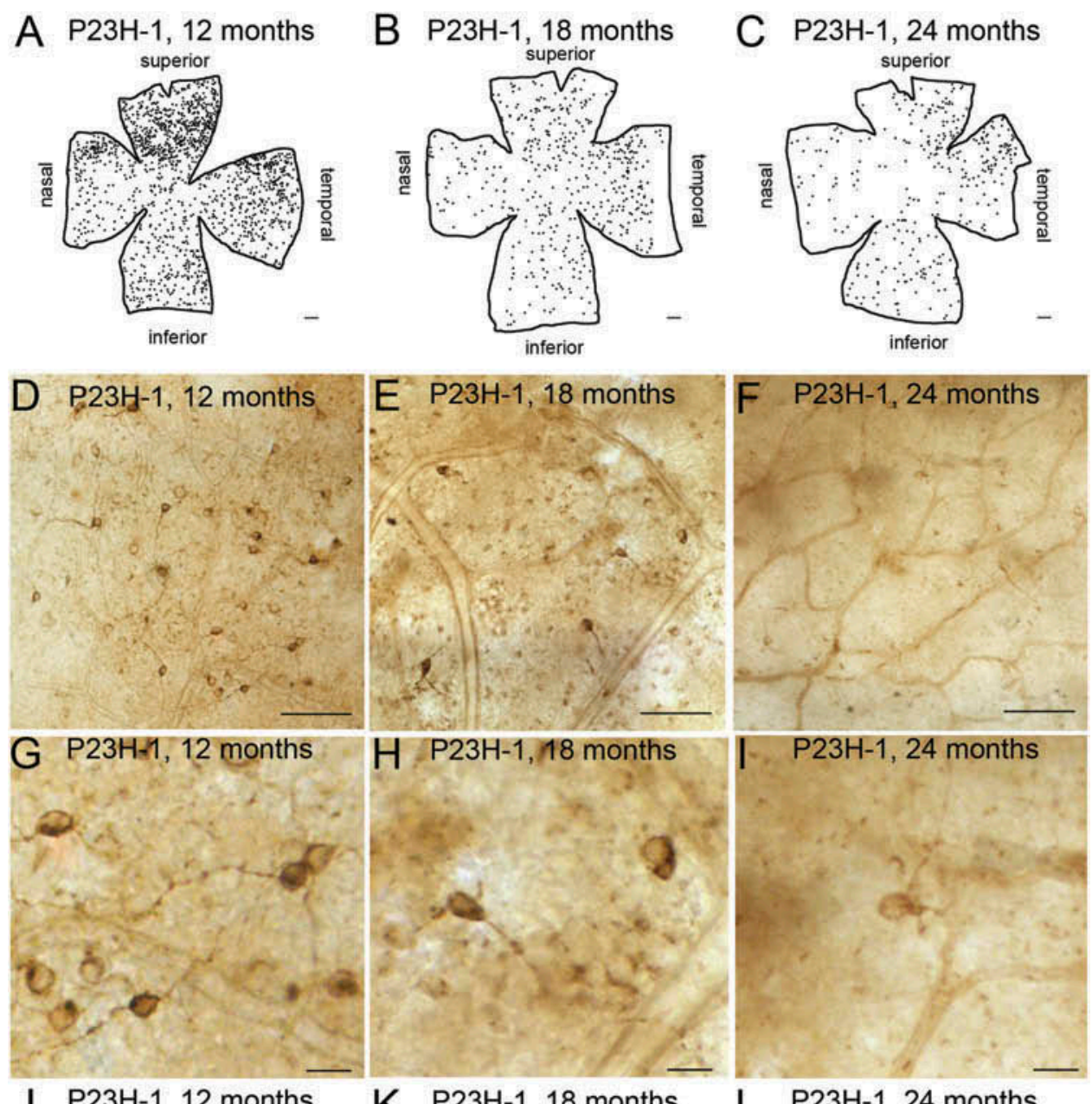

J $\mathrm{P} 23 \mathrm{H}-1,12$ months
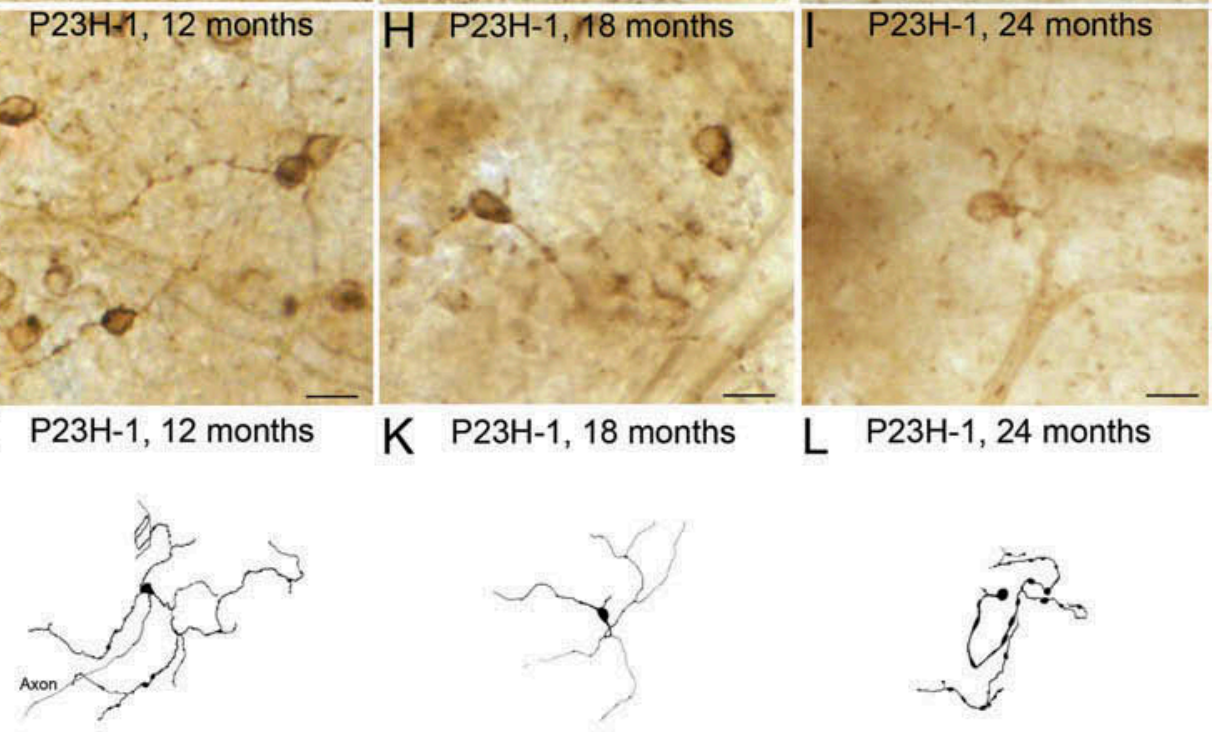

Figure 2. Distribution, morphology and arborization of $m R G C s$ in $\mathrm{P} 23 \mathrm{H}-1$ rat retinas. $(\mathrm{A}-\mathrm{C})$ Representative drawings of whole-mount retinas from $\mathrm{P} 23 \mathrm{H}-1$ rats at $12(\mathrm{~A}), 18(\mathrm{~B})$ and 24 (C) months of age. Retinas were drawn by hand, showing the location of individual mRGCs labeled with immunoperoxidase. (D-F) Examples of mRGCs in whole-mount rat retinas from P23H-1 rats at 12 (D), 18 (E) and 24 (F) months of age. Each image is the projection of 5-10 focal planes between the GCL and the stratum S1 of the IPL. (G-I) High magnification of D-F, respectively. (J-L) Representative drawings of the soma and complete dendritic field of mRGCs from a region of the central retina (between the superior and nasal quadrants) of $\mathrm{P} 23 \mathrm{H}-1$ rats at $12(\mathrm{~J}), 18(\mathrm{~K})$ and $24(\mathrm{~L})$ months of age. Scale bars: A-C, $500 \mu \mathrm{m}$; D-F, $100 \mu \mathrm{m} ; \mathrm{G}-\mathrm{I}, 20 \mu \mathrm{m} ; \mathrm{J}-\mathrm{L}, 50 \mu \mathrm{m}$.

cells $/ \mathrm{mm}^{2}, 552 \pm 74$ cells/retina, $n=3$; Figure 3 ) was similar to that obtained at 18 months of age.

The spatial distribution and number of melanopsin-containing ganglion cells was also evaluated in 30-month-old $\mathrm{P} 23 \mathrm{H}-1$ rats. The spatial distribution of mRGCs showed a similar arrangement to that observed in younger animals

(Figure 4). The mean number of mRGCs $(3.3 \pm 1.2$ cells $/ \mathrm{mm}^{2}, 129 \pm 102$ cells/retina, $n=3$ ) was significantly lower than that measured in 12-monthold $\mathrm{P} 23 \mathrm{H}-1$ rats (ANOVA, Bonferroni's test, $P$ $<0.05)$. 


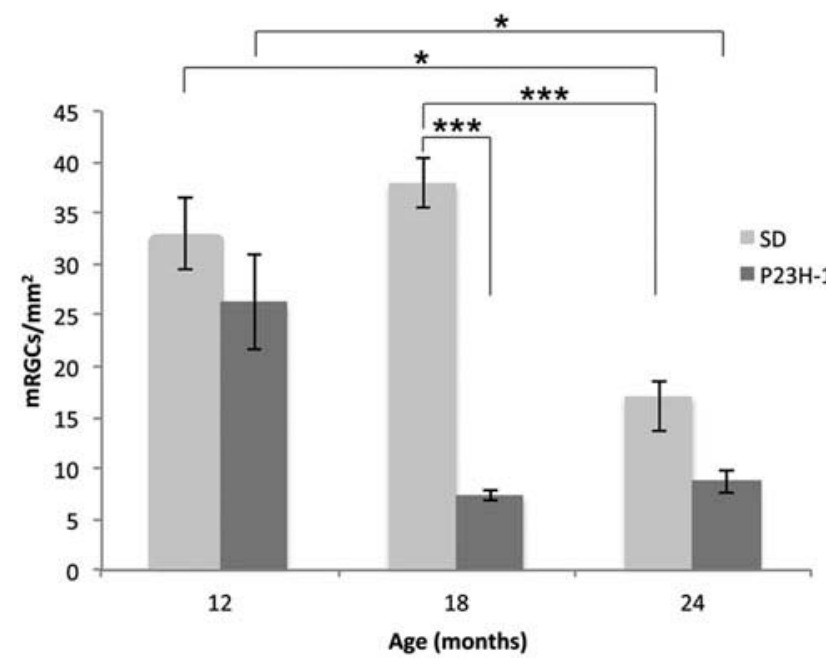

Figure 3. Mean density of mRGCs in control Sprague-Dawley and P23H-1 rats. Quantification of the mean density (cells $/ \mathrm{mm}^{2}$ ) of mRGCs in Sprague-Dawley (SD) rats at $12(n=7), 18(n=7)$ and 24 $(n=4)$ months of age, and $\mathrm{P} 23 \mathrm{H}-1$ rats aged $12(n=3), 18(n=6)$ and $24(n=3)$ months. Each of the cell bodies labeled with immunoperoxidase was counted in each entire retina examined. ANOVA, Bonferroni's test, ${ }^{* *} P<0.05,{ }^{* * *} P<0.001$.

\section{Progressive degeneration of $m R G C$ structure in P23H-1 rats}

To analyze mRGC neurite morphology, we manually traced the outlines of the cell body and the complete dendritic field of representative melanopsin-positive cells. In control Sprague-Dawley rats, mRGC dendrites formed a dense plexus in the inner plexiform layer at all three ages tested (Figure 1J-L). However, a progressive age-dependent decrease in the dendritic area and the number of branch points of mRGCs was observed in $\mathrm{P} 23 \mathrm{H}-1$ rats (Figure 2J-L), indicating a gradual degeneration associated with age. At 30 months of age, mRGCs in $\mathrm{P} 23 \mathrm{H}-1$ rats were very few and scattered, with extremely short and limited dendrites (Figure 4).
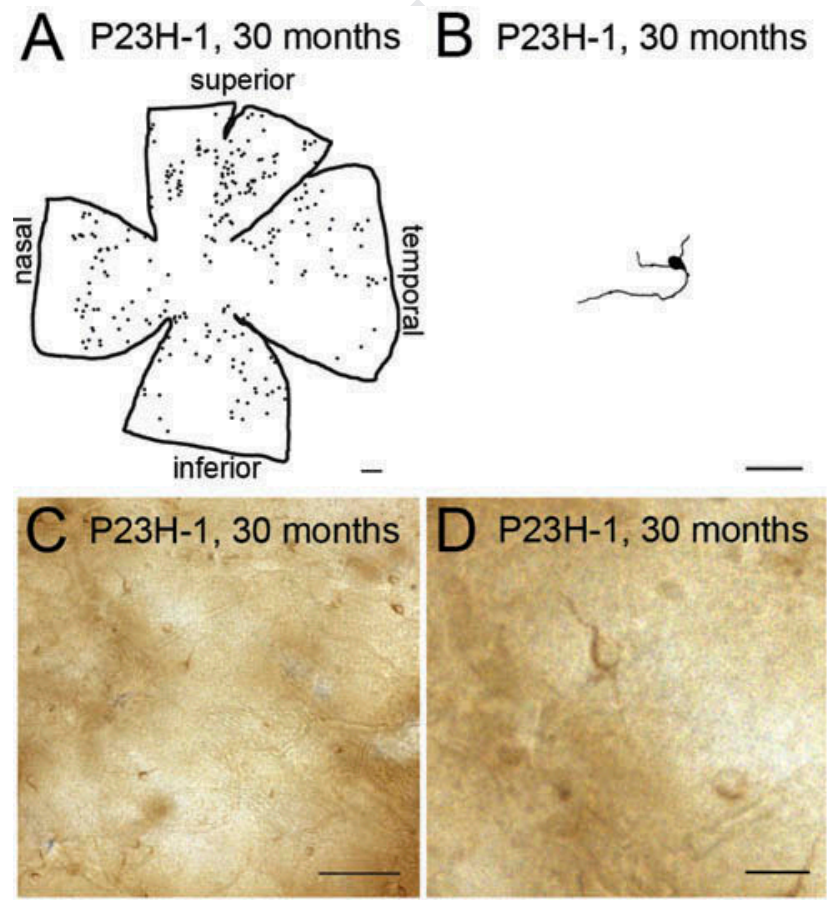

Figure 4. Distribution, morphology and arborization of mRGCs in 30-month-old P23H-1 rats. (A) Representative drawings of a whole-mount retina from a $\mathrm{P} 23 \mathrm{H}-1$ rat at 30 months of age. The retina was drawn by hand, showing the location of individual mRGCs labeled with immunoperoxidase. (B) Representative drawings of the soma and complete dendritic field of an mRGCs from a region of the central retina (between the superior and nasal quadrants) of a P23H-1 rat at 30 months of age. (C, D) Examples of mRGCs in whole-mount rat retinas from $\mathrm{P} 23 \mathrm{H}-1$ rats at 30 months of age. Each image is the projection of 5 to 10 focal planes between the GCL and the stratum S1 of the IPL. Scale bars: A, $500 \mu \mathrm{m} ; \mathrm{B} 50 \mu \mathrm{m} ; \mathrm{C}, 100 \mu \mathrm{m} ; \mathrm{D}, 20 \mu \mathrm{m}$. 
A
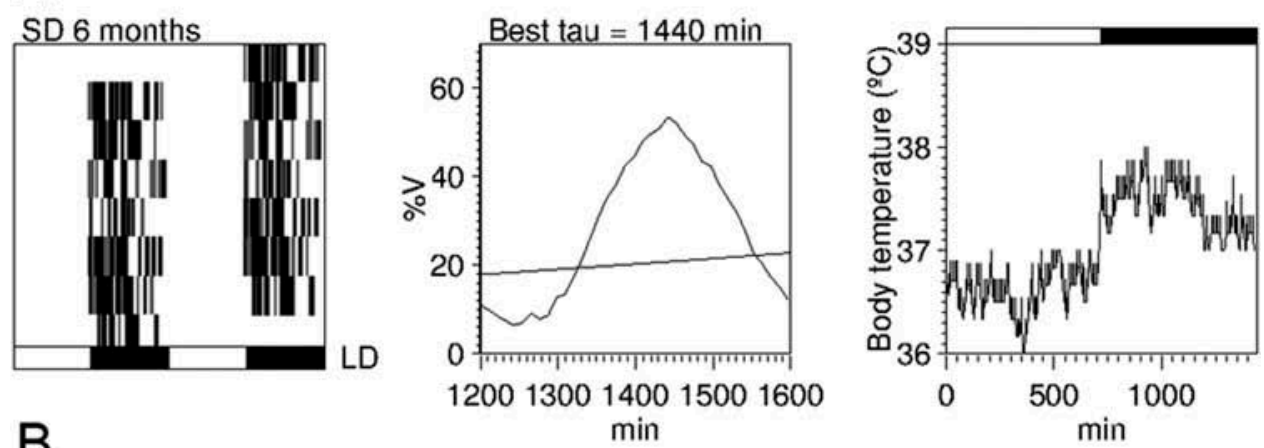

B
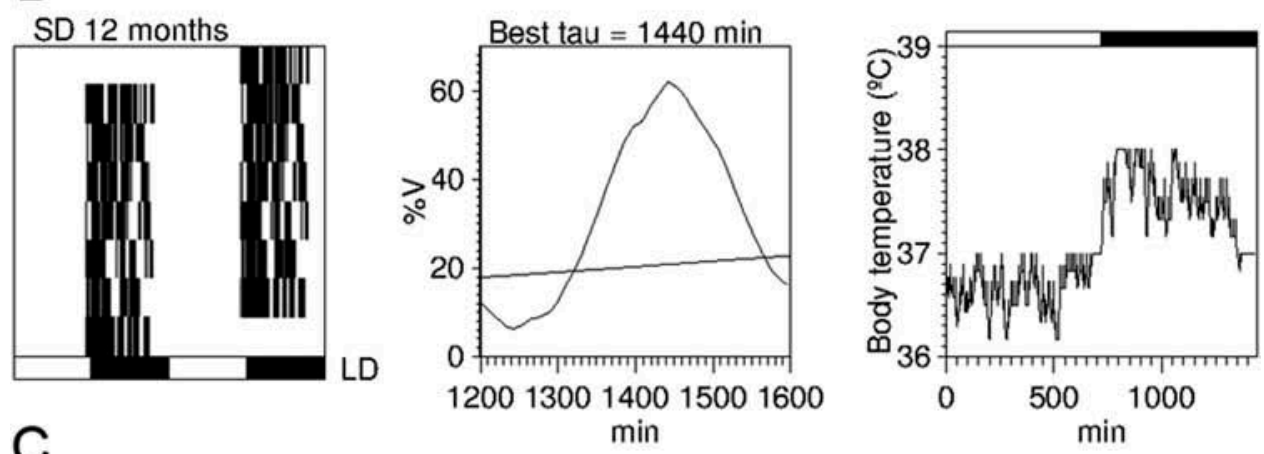

C
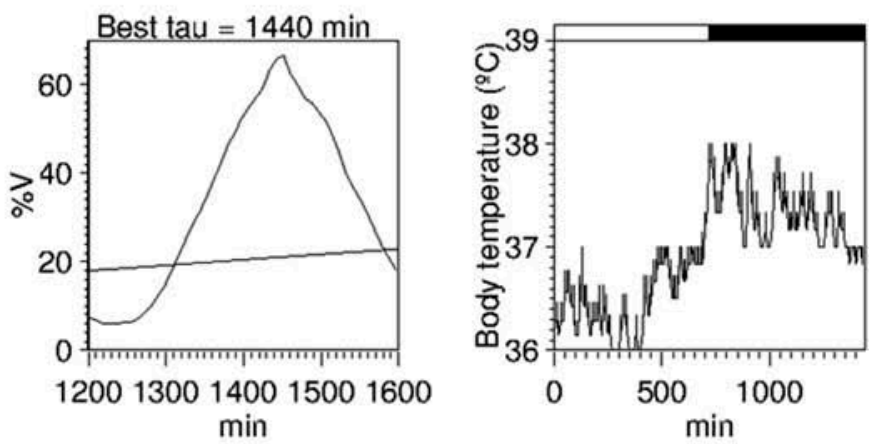

SD 24 months
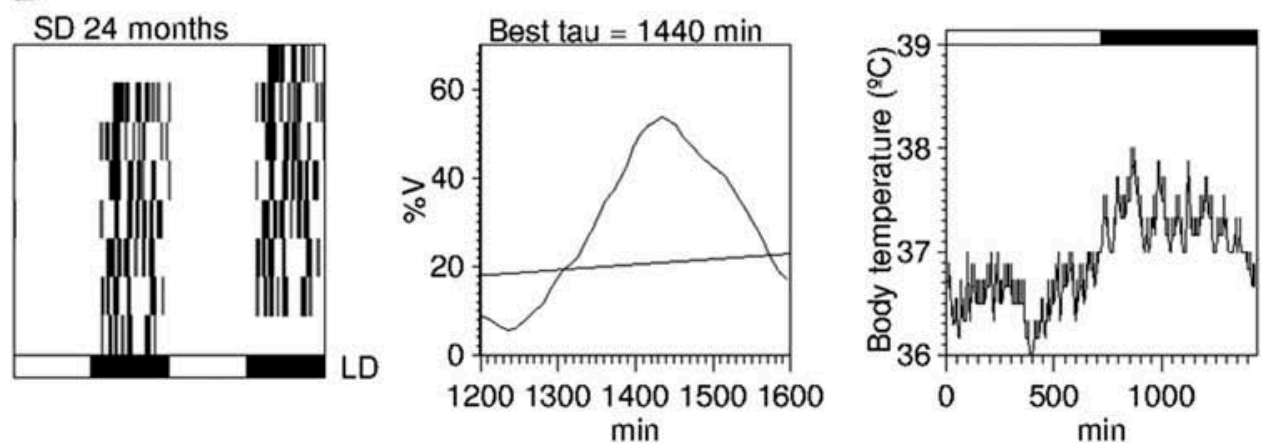

Figure 5. Circadian rhythms of core-body temperature in Sprague-Dawley rats. Representative actograms (left panels), periodograms (middle panels), and mean waveforms (right panels) at the ages of 6 (A), 12 (B), 18 (C), and 24 (D) months for a control Sprague-Dawley (SD) rat exposed to a 12:12 LD cycle. All data were obtained from the same animal. Light and dark schedules are represented by white and dark bars, respectively.

\section{Age-related changes in body temperature rhythms in control and $\mathrm{P} 23 \mathrm{H}-1$ rats}

To assess circadian rhythms and photoentrainment in control Sprague-Dawley and P23H-1 rats, the core-body temperature of each animal was continuously monitored from 2 to 24 months of age. Figures 5 and 6 show representative actograms, Sokolove-Bushell periodograms and mean waveforms for body temperature from a control 
A

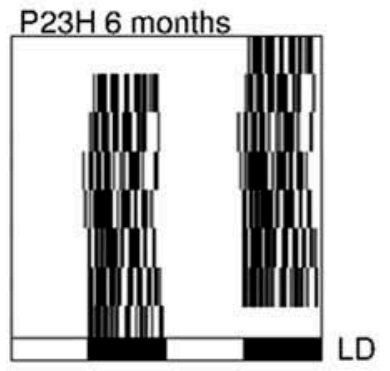

B

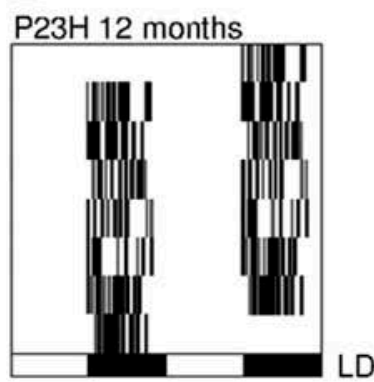

C

$\mathrm{P} 23 \mathrm{H} 18$ months

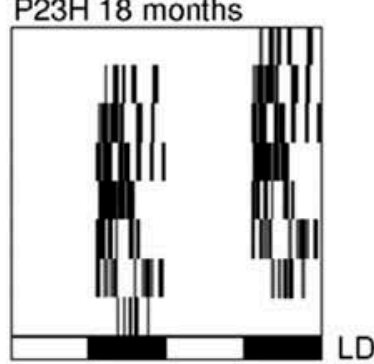

D

P23H 24 months

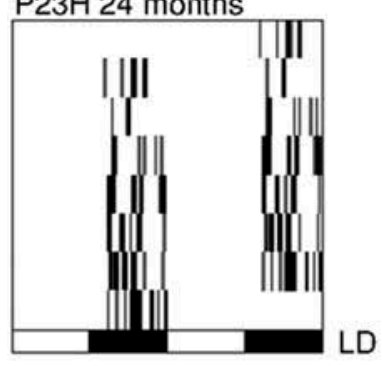

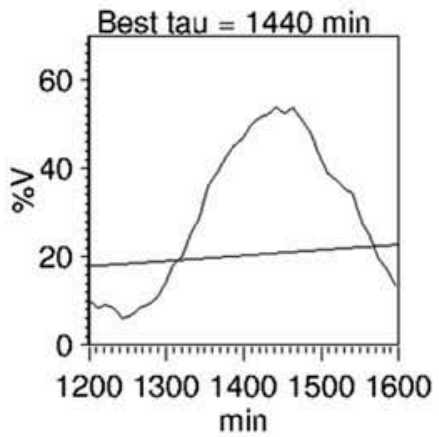
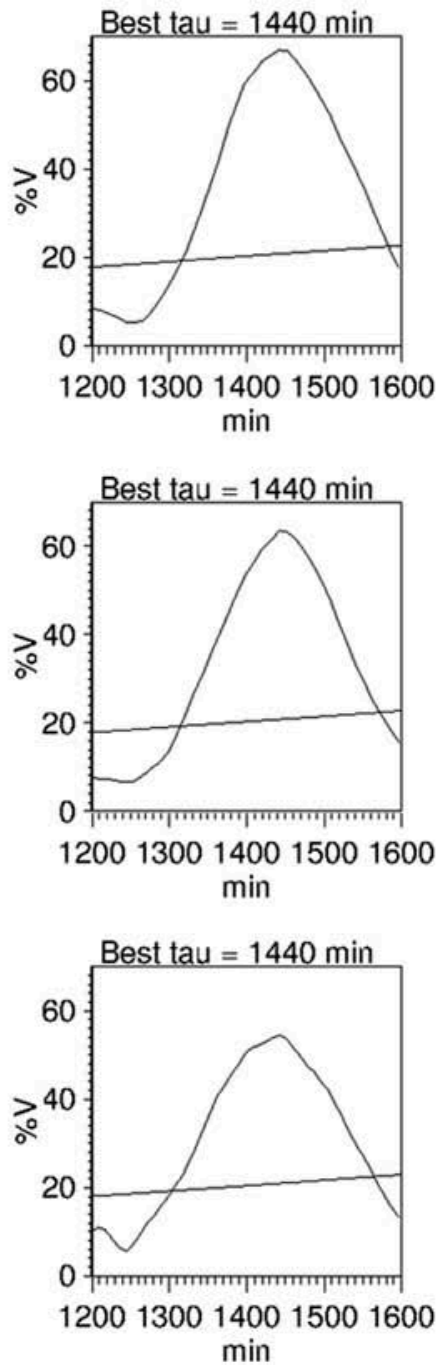
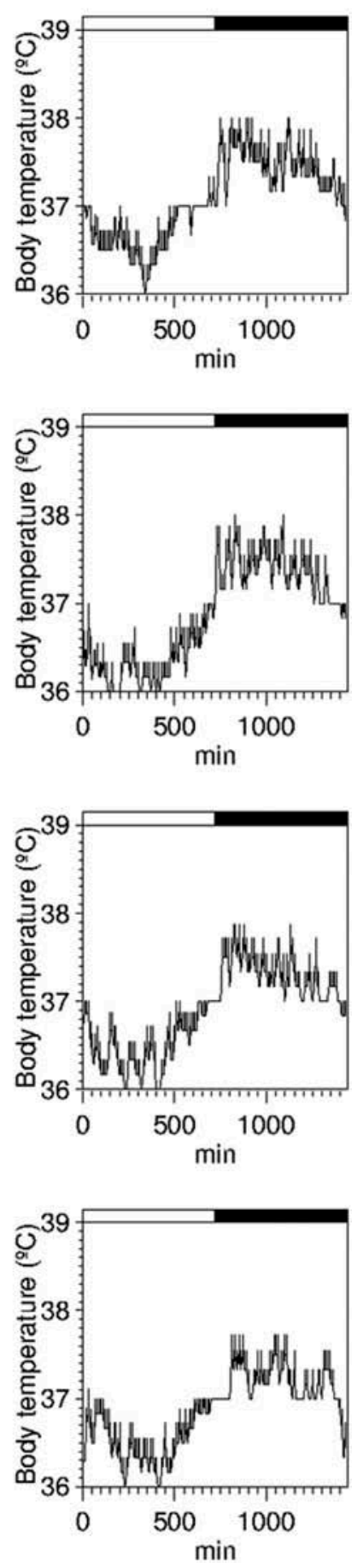

Figure 6. Circadian rhythms of core-body temperature in $\mathrm{P} 23 \mathrm{H}-1$ rats. Example of actograms (left panels), periodograms (middle panels), and mean waveforms (right panels) at the ages of 6 (A), 12 (B), 18 (C), and 24 (D) months for a P23H-1 rat exposed to a 12:12 LD cycle. All data were obtained from the same animal. Light and dark schedules are represented by white and dark bars, respectively.

(Figure 5) and a $\mathrm{P} 23 \mathrm{H}-1$ rat (Figure 6) at 6, 12, 18, and 24 months of age. Throughout the experiment, body temperature exhibited a robust circadian rhythm in control animals, regardless of the age or strain of the animal, with no differences in the periodogram or in the pattern of the mean waveform, even at 24 months of age. However, body temperature circadian rhythms were less 

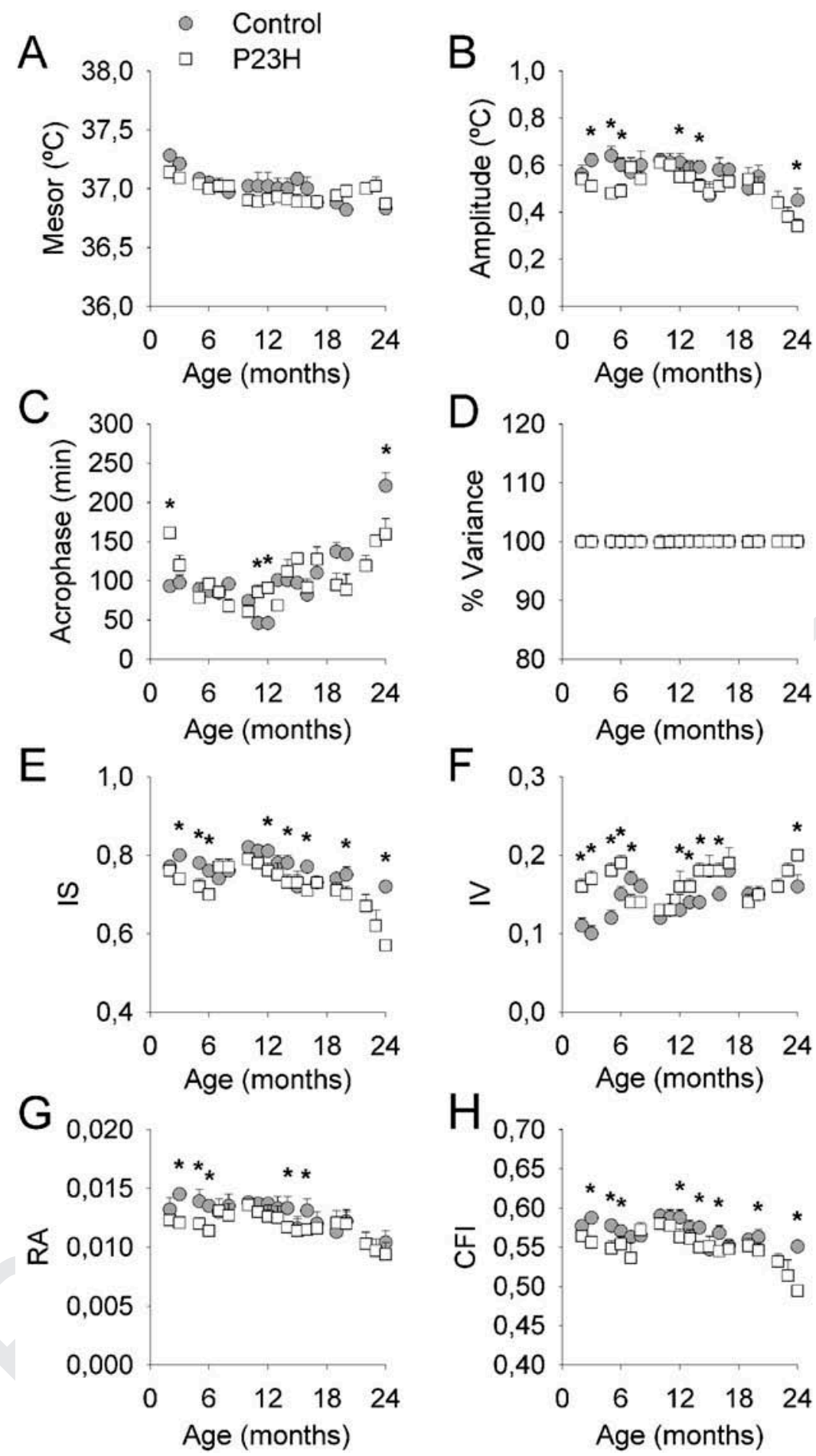

Figure 7. Age-related changes in circadian core-body temperature parameters in Sprague-Dawley and $\mathrm{P} 23 \mathrm{H}-1$ rats. Rhythm parameters (left panels) and non-parametric variables (right panels) from control Sprague-Dawley (SD) $(n=9)$ and P23H-1 $(n=$ 14) rats throughout their lifetime. Mesor $=24$-h time series mean; amplitude = one-half the peak-to-trough variation of the $24-h$ rhythm; acrophase = peak time relative to local midnight; \% variance = index of goodness of fit of the 24-h waveform approximation to the time series data; IS = interdaily stability; IV = intradaily variability; RA = relative amplitude; CFI = circadian function index. Repeated measures ANOVA, Bonferroni's test, ${ }^{*} P<0.05$.

robust throughout the experiment in $\mathrm{P} 23 \mathrm{H}-1$ rats. The mesor (24-h time series mean) values from the body temperature records were lower within age in $\mathrm{P} 23 \mathrm{H}-1$ rats (repeated measures ANOVA, Bonferroni's test, $P<0.001$; Table 1, Figure 7A), but there were no differences between genotypes. 
A

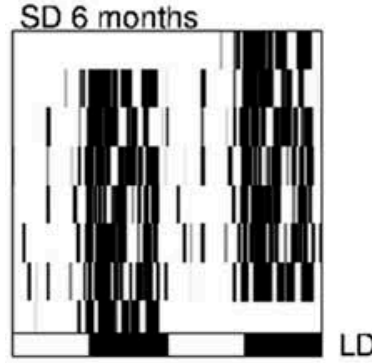

B

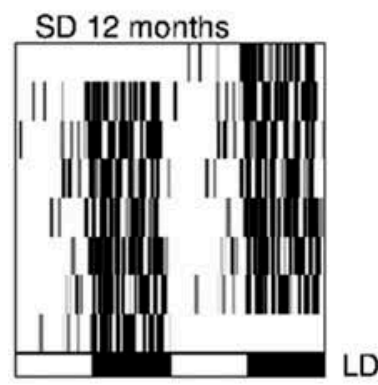

C

SD 18 months

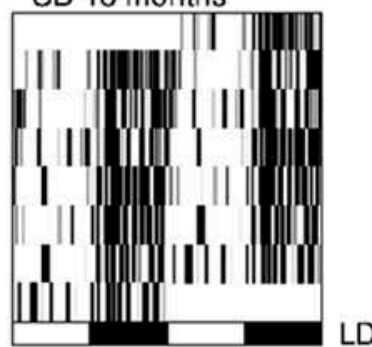

$\mathrm{D}$

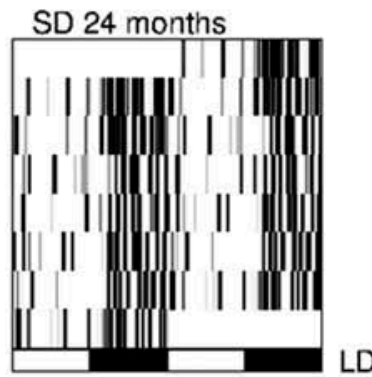

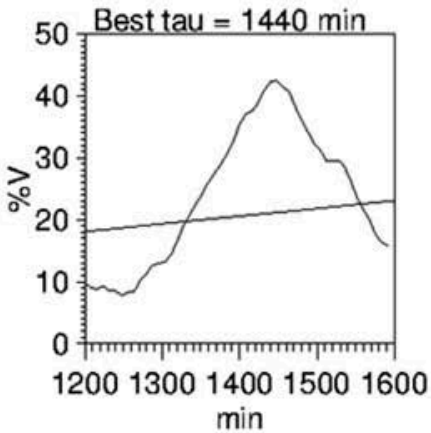
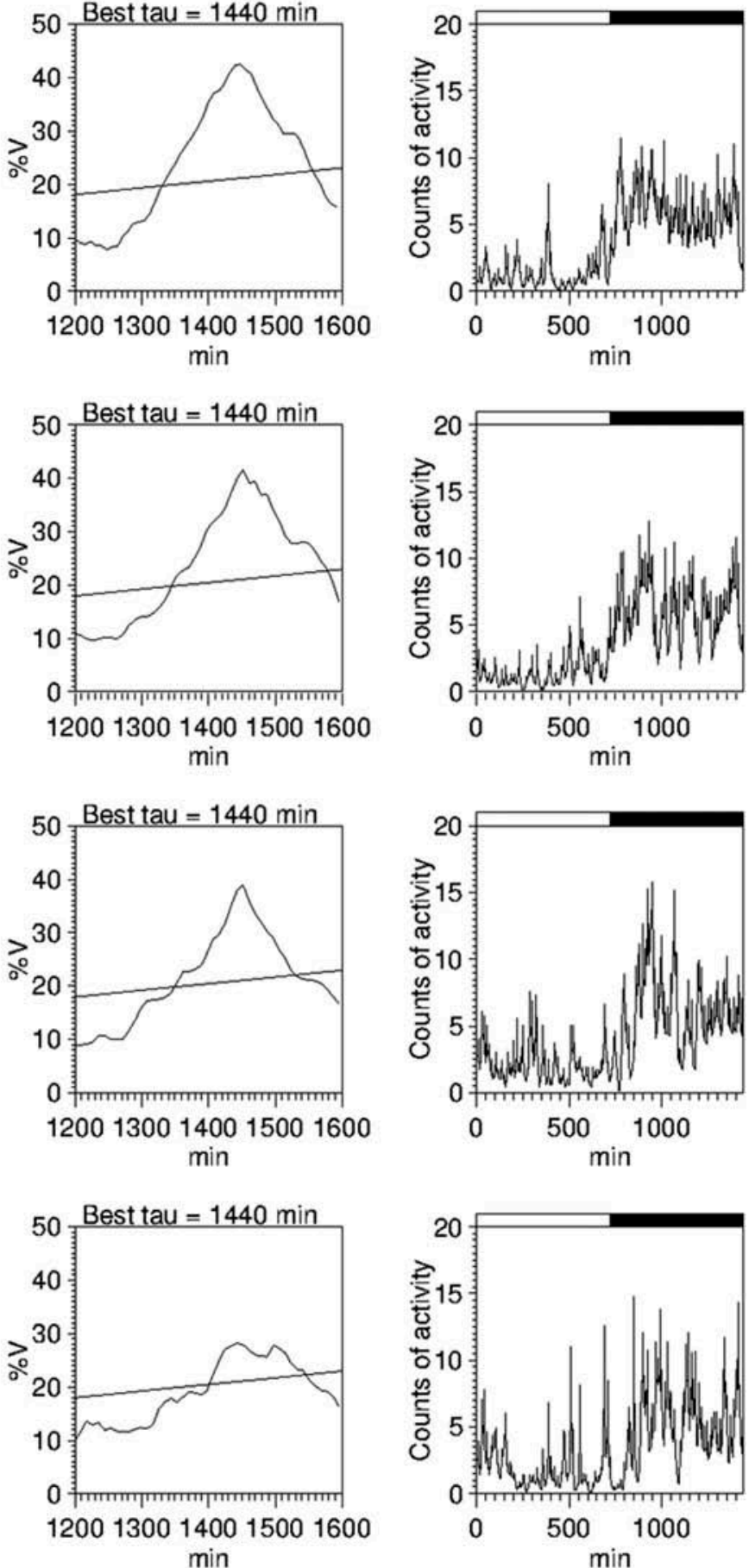

Figure 8. Circadian rhythms of locomotor activity in Sprague-Dawley rats. Representative actograms (left panels), periodograms (middle panels), and mean waveforms (right panels) at the ages of 6 (A), 12 (B), 18 (C), and 24 (D) months for a Sprague-Dawley (SD) rat exposed to a 12:12 LD cycle. All data were obtained from the same animal. Light and dark schedules are represented by white and dark bars, respectively.

However, significant differences were found throughout the experiment between control and $\mathrm{P} 23 \mathrm{H}-1$ rats in terms of either the amplitude (one-half the peak-to-trough variation of the 24- h rhythm, Figure 7B) or the acrophase (peak time relative to local midnight of the temporal pattern, as derived by the 24 -h waveform approximation; Figure 7C) (repeated measures ANOVA, 
A
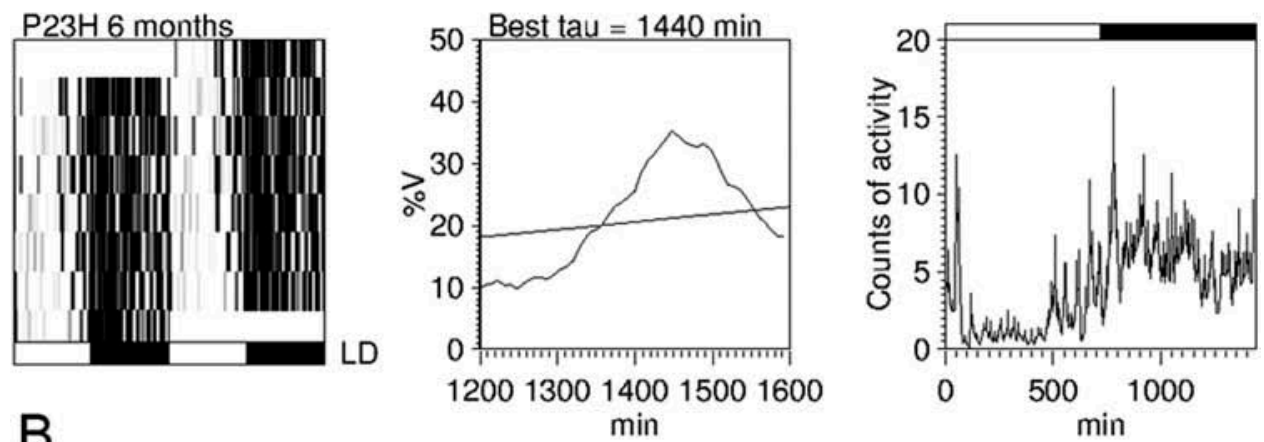

B
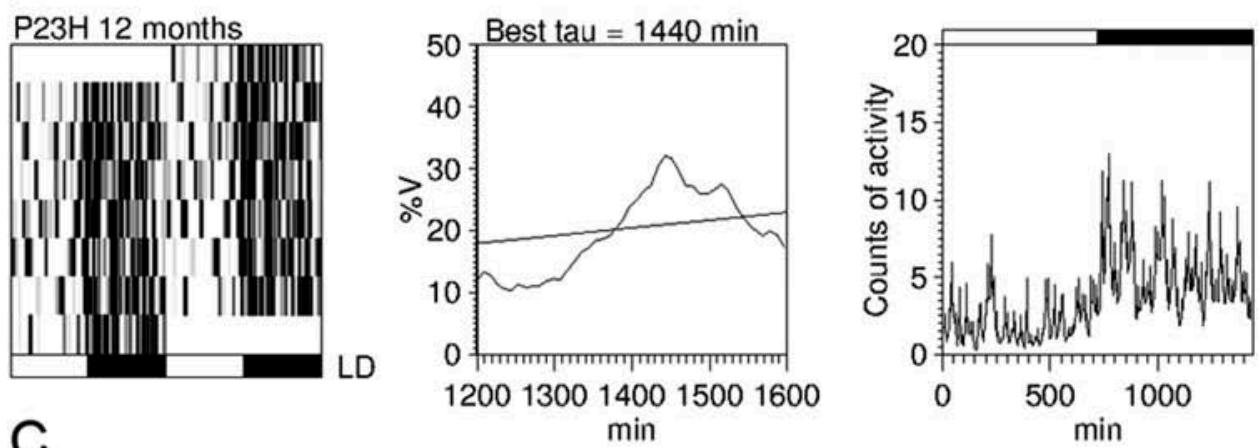

C
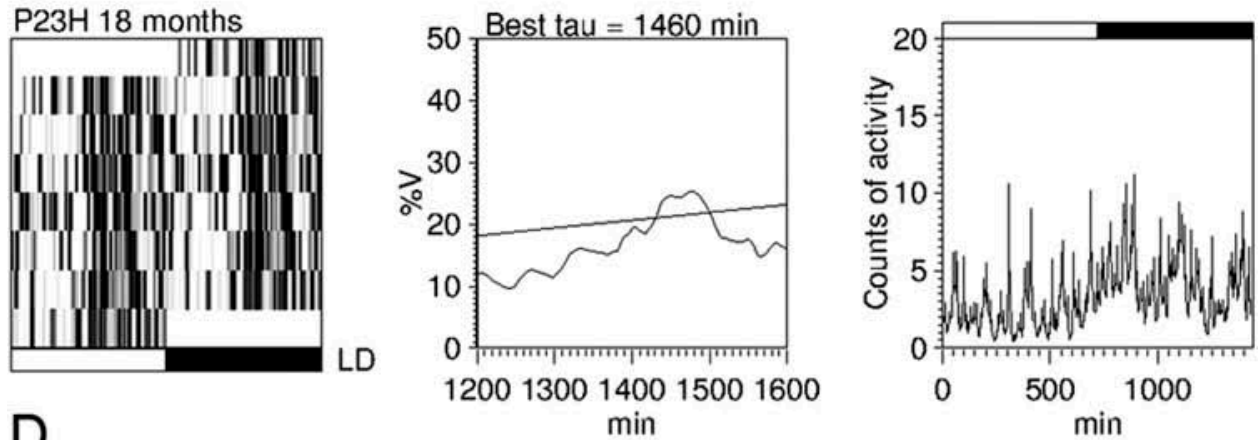

D
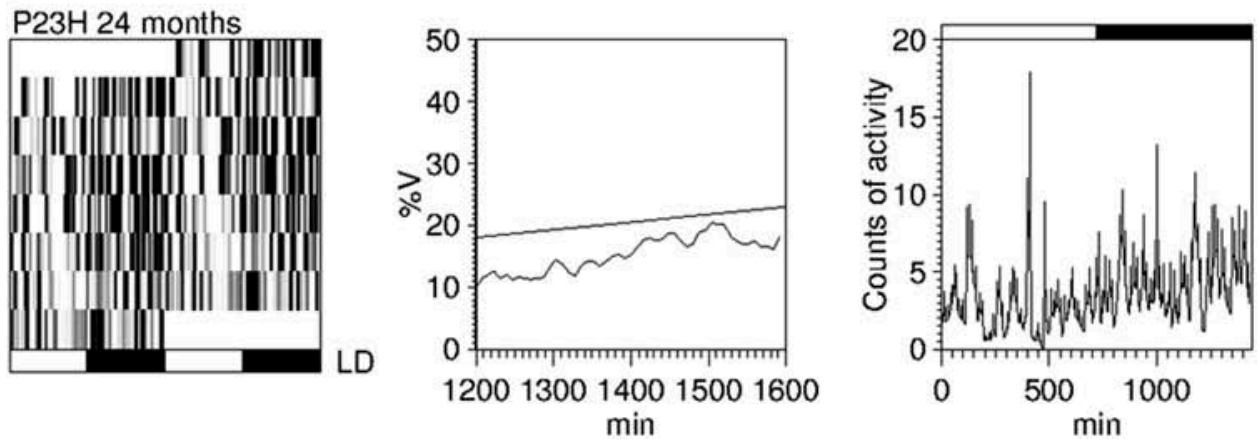

Figure 9. Circadian rhythms of locomotor activity in $\mathrm{P} 23 \mathrm{H}-1$ rats. Example of actograms (left panels), periodograms (middle panels), and mean waveforms (right panels) at the ages of 6 (A), 12 (B), 18 (C), and 24 (D) months for a P23H-1 rat exposed to a 12:12 LD cycle. All data were obtained from the same animal. Light and dark schedules are represented by white and dark bars, respectively.

Bonferroni's test, genotype effect $P<0.05$ in both 415 cases; Table 1).
To better understand the effects of visual degeneration on the circadian patterns of body temperature, we evaluated the degree of phase homogeneity in control and $\mathrm{P} 23 \mathrm{H}-1$ rats by means of non-parametric variables. The coupling strength of the body temperature rhythm to 

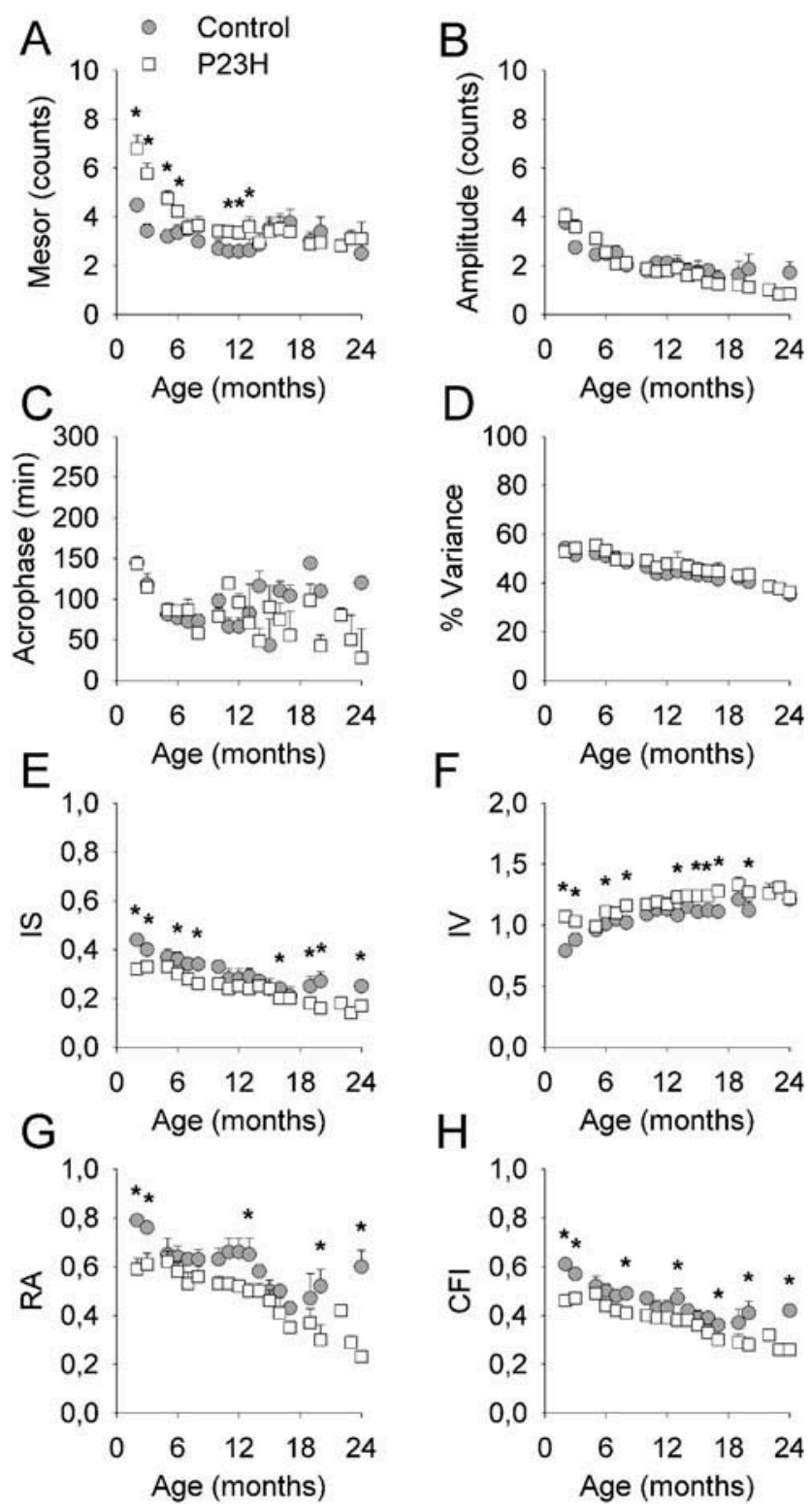

Figure 10. Age-related changes in circadian locomotor activity parameters in Sprague-Dawley and P23H-1 rats. Rhythm parameters (left panels) and non-parametric variables (right panels) from control Sprague-Dawley (SD) $(n=9)$ and P23H-1 $(n=14)$ rats throughout their lifetime. Mesor = 24-h time series mean; amplitude = one-half the peak-to-trough variation of the 24-h rhythm; acrophase $=$ peak time relative to local midnight; \% variance $=$ index of goodness of fit of the 24-h waveform approximation to the time series data; IS = interdaily stability; IV = intradaily variability; RA = relative amplitude; $C F I$ = circadian function index. Repeated measures ANOVA, Bonferroni's test, ${ }^{*} P<0.05$.

environmental zeitgebers (IS) was significantly weaker in $\mathrm{P} 23 \mathrm{H}-1$ rats than in control rats, with higher differences in the last phase of the experiment (repeated measures ANOVA, age effect $P<$ 0.001 , genotype effect $P<0.001$; Table 1 , Figure 7E). The analysis of the body temperature rhythm also revealed a significantly higher fragmentation, measured as IV (repeated measures ANOVA, genotype effect, $P<0.05$; Table 1 ,
Figure $7 \mathrm{~F}$ ), and a decrease in the RA (repeated measures ANOVA, genotype effect $P<0.05$; Table 1, Figure 7G) in $\mathrm{P} 23 \mathrm{H}-1$ rats as compared to control animals. The CFI, a parameter that takes into account RA, IS, and IV, also revealed a lower robustness of the circadian pattern as recorded in $\mathrm{P} 23 \mathrm{H}-1$ versus control rats (repeated measures ANOVA, genotype effect $P<0.0001$; Table 1 , Figure $7 \mathrm{H})$. 
Table 1. Circadian parameters.

\begin{tabular}{|c|c|c|c|c|c|c|}
\hline & \multicolumn{3}{|c|}{ Body Temperature } & \multicolumn{3}{|c|}{ Locomotor Activity } \\
\hline & Control $n=9$ & $\mathrm{P} 23 \mathrm{H}-1 n=14$ & $\begin{array}{c}\text { Correlation with mRGC } \\
\text { Density }\end{array}$ & $\begin{array}{c}\text { Control } n= \\
9\end{array}$ & $\begin{array}{c}\mathrm{P} 23 \mathrm{H}-1 n= \\
14\end{array}$ & $\begin{array}{c}\text { Correlation with mRGC } \\
\text { Density }\end{array}$ \\
\hline \multicolumn{7}{|l|}{ Rhythm parameters } \\
\hline Mesor ( ${ }^{\circ} \mathrm{C} /$ counts) & $37.02 \pm 0.02$ & $36.96 \pm 0.01$ & -0.140 & $3.16 \pm 0.10$ & $3.88 \pm 0.09^{*}$ & -0.134 \\
\hline $\begin{array}{l}\text { Amplitude }\left({ }^{\circ} \mathrm{C} /\right. \\
\text { counts) }\end{array}$ & $0.57 \pm 0.01$ & $0.48 \pm 0.01^{*}$ & 0.244 & $2.11 \pm 0.07$ & $1.83 \pm 0.07$ & 0.360 \\
\hline Acrophase (min) & $99.67 \pm 9.52$ & $104.55 \pm 6.98^{*}$ & $-0.475^{*}$ & $95.67 \pm 6.91$ & $87.82 \pm 10.51$ & 0.011 \\
\hline $\begin{array}{l}\% \text { Variance } \\
\text { accounted }\end{array}$ & $99.99 \pm 0.005$ & $99.98 \pm 0.004$ & -0.288 & $45.64 \pm 0.60$ & $45.95 \pm 0.55$ & 0.013 \\
\hline \multicolumn{7}{|l|}{ Non-parametric variables } \\
\hline IS & $0.77 \pm 0.006$ & $0.68 \pm 0.005^{* * *}$ & $0.627^{*}$ & $0.30 \pm 0.01$ & $0.23 \pm 0.01^{*}$ & 0.203 \\
\hline IV & $0.14 \pm 0.003$ & $0.16 \pm 0.003^{*}$ & $-0.601^{*}$ & $1.07 \pm 0.01$ & $1.19 \pm 0.01^{*}$ & $-0.561^{*}$ \\
\hline RA & $0.013 \pm 0.000$ & $0.011 \pm 0.000^{*}$ & $0.587^{*}$ & $0.60 \pm 0.01$ & $0.45 \pm 0.01^{*}$ & $0.416^{*}$ \\
\hline CFI & $0.57 \pm 0.002$ & $0.54 \pm 0.002^{* * *}$ & $0.674^{*}$ & $0.46 \pm 0.01$ & $0.36 \pm 0.01^{*}$ & $0.497^{*}$ \\
\hline
\end{tabular}

Circadian parameters of body temperature and locomotor activity (mean \pm SEM) from control Sprague-Dawley and P23H-1 rats, and Pearson's product moment correlation coefficients from the correlational analyses between mRGC density and circadian parameters. IS, interdaily stability; IV, intradaily variability; RA, relative amplitude; $\mathrm{CFI}$, circadian function index; $\mathrm{mRGC}$, melanopsin-expressing retinal ganglion cells. Repeated measures ANOVA, genotype effect (P23H vs. Control): ${ }^{*} P<0.05,{ }^{* *} P<0.001,{ }^{* * *} P<0.0001$; Pearson's product moment correlation coefficients (mRGC density vs circadian parameters): ${ }^{*} P<0.05$.

\section{Age-related changes in locomotor activity rhythms in control and $\mathrm{P} 23 \mathrm{H}-1$ rats}

We also recorded locomotor activity in control Sprague-Dawley and $\mathrm{P} 23 \mathrm{H}-1$ rats from 2 to 24 months of age. Figures 8 and 9 show representative locomotor activity actograms, Sokolove-Bushell periodograms and mean waveforms from a control (Figure 8) and a $\mathrm{P} 23 \mathrm{H}-1$ rat (Figure 9) exposed to a 12:12 LD cycle at the ages of $6,12,18$, and 24 months. In control rats, locomotor activity exhibited a robust circadian rhythm throughout the experiment, up to 18 months of age, with no differences in the actogram patterns, periodograms or mean daily waveforms. During the final experimental period, however, we observed a slight deterioration in the circadian locomotor activity rhythms, with weak circadian oscillations in actograms and mean waveforms, and low significance of peaks in the periodograms at 24 months of age (Figure 8D).

In $\mathrm{P} 23 \mathrm{H}-1$ rats, the locomotor activity circadian

460 rhythm showed a progressive age-related deterioration throughout the experiment, with a gradual weakness in the rest-activity cycle in the actograms and mean waveforms (Figure 9). Moreover, in $\mathrm{P} 23 \mathrm{H}-1$ rats, the Sokolove-Bushell 465 periodogram analysis of locomotor activity showed no significant peaks at 24 months of age. The mesor values were higher in $\mathrm{P} 23 \mathrm{H}-1$ versus control animals (repeated measures ANOVA, genotype effect $P<0.05$; Table 1, Figure 10A). But no differences were found between control and $\mathrm{P} 23 \mathrm{H}-1$ rats in terms of the amplitude (Figure 10B), acrophase (Figure 10C) or the percentage of variance accounted for by the locomotor activity rhythms (Figure 10D).

The analysis of non-parametric variables in the locomotor activity records also evidenced that locomotor activity circadian rhythms were less robust throughout the experiment in $\mathrm{P} 23 \mathrm{H}-1$ rats, as compared to the control animals. The coupling strength of the locomotor activity rhythm to environmental zeitgebers (IS), the RA of the rhythm (RA), and the CFI were significantly lower in $\mathrm{P} 23 \mathrm{H}-1$ rats than in control rats (repeated measures ANOVA, genotype effect $P<0.05$ in all cases; Table 1, Figure 10E, 10G, 10H, respectively). On the contrary, the locomotor activity rhythm revealed a significantly higher IV (repeated measures ANOVA, genotype effect $P<0.05$; Table 1 , Figure 10F) in $\mathrm{P} 23 \mathrm{H}-1$ rats as compared to control animals, also indicating a less robust circadian pattern in $\mathrm{P} 23 \mathrm{H}-1$ versus control rats.

When age-related changes in mRGCs were compared to the results obtained from the chronobiological evaluation, a correlation was found between mRGC density and circadian rhythm parameters. In fact, the mRGC density values from control and $\mathrm{P} 23 \mathrm{H}-1$ rats positively correlated 
with the body temperature IS, RA, and CFI ( $R=$ $0.627,0.587$, and 0.674 , respectively; $P<0.05)$ and

with the locomotor activity RA and CFI $(R=0.416$ and 0.497 , respectively; $P<0.05)$. mRGC density correlated negatively with the body temperature acrophase and IV $(R=-0.475$ and -0.601 , respectively; $P<0.05)$ and with the locomotor activity IV $(R=0.561 ; P<0.05)$.

\section{Discussion}

The present work provides evidence that RP in $\mathrm{P} 23 \mathrm{H}-1$ rats is associated with both a progressive degeneration of mRGCs and a gradual deteriora-

510 tion of the circadian system. The progressive loss in density, integrity, and dendritic arborization of mRGCs in advanced stages of the degenerative disease correlates with decreased RAs, weaker coupling strength of the rhythm to environmental zeitgebers and higher rhythm fragmentation in $\mathrm{P} 23 \mathrm{H}-1$ rats. In previous studies, we have demonstrated that retinal degeneration positively correlates with the occurrence of circadian dysfunctions in P23H-3 rats (Lax et al., 2011), and that mela-

520 nopsin-positive ganglion cells degenerate in advanced stages of the disease (Esquiva et al., 2013; Garcia-Ayuso et al., 2015). However, to our knowledge, this is the first longitudinal study that jointly examines the effects of retinal degeneration and aging on both the melanopsin system and circadian photoentrainment.

The present study concerns $\mathrm{P} 23 \mathrm{H}$ rats, which have been engineered to mimic a naturally occurring mutation in $\mathrm{P} 23 \mathrm{H}$ patients (Cuenca et al., 530 2004; Machida et al., 2000; Pinilla et al., 2005). In $\mathrm{P} 23 \mathrm{H}$ transgenic albino rats, both line 1 (faster degeneration) and line 3 (slower degeneration) suffer from a progressive loss of photoreceptors, accompanied by degenerative changes in the inner 535 retina (Cuenca et al., 2014; Cuenca et al., 2004; Marc et al., 2003; Puthussery \& Taylor, 2010) and a substantial degeneration of retinal ganglion cells (Garcia-Ayuso et al., 2010; Kolomiets et al., 2010). In this study, we used $\mathrm{P} 23 \mathrm{H}-1$ transgenic albino 540 rats in order to obtain a high degree of retinal degeneration at each age tested. Previous studies on $\mathrm{P} 23 \mathrm{H}-1$ rats have shown that intensity response electroretinograms are already severely depressed at P21 as compared to age-matched Sprague-
Dawley rats (Pinilla et al., 2005). Furthermore, 545 only sporadic photoreceptors have been found in 9-month-old $\mathrm{P} 23 \mathrm{H}-1$ rats (Cuenca et al., 2004), and a total loss of rod and cone photoreceptors has been described in $\mathrm{P} 23 \mathrm{H}-1$ rats aged 12 months and older (Pinilla et al., 2015). In our results, $\mathrm{P} 23 \mathrm{H}-1$ animals showed a progressive age-related degeneration of mRGCs, which is in accordance with data previously described for $\mathrm{P} 23 \mathrm{H}-3$ rats (Esquiva et al., 2013; Garcia-Ayuso et al., 2015).

Since the daily light-dark cycle is the primary environmental zeitgeber in mammals, via the mRGCs, ocular pathologies have been classically associated with circadian disorders. Many studies have correlated altered circadian rhythms with inner retinal diseases, such as glaucoma (de Zavalia et al., 2011; Jean-Louis et al., 2008), diabetic retinopathy (Fernandez et al., 2013; Lahouaoui et al., 2014), or retinal ischemia (Gonzalez Fleitas et al., 2015). But circadian disorders have also been described in advanced stages of diseases of the outer retina, including RP. Accordingly, it has been shown that sleep quality decreases in RP patients in an age-dependent manner (Gordo et al., 2001; Ionescu et al., 2001), and alterations in blood pressure and heart rate circadian rhythmic structure have been demonstrated in non-blind patients affected by RP (Cugini et al., 2001). Moreover, circadian dysfunctions have been shown in $\mathrm{rds} / \mathrm{rds}$ mice (Mrosovsky \& Thompson, 2008) and P23H-3 rats (Lax et al., 2011), which are both animal models of RP. In agreement with this, $\mathrm{P} 23 \mathrm{H}-1$ rats showed impaired circadian rhythmicity in body temperature and locomotor activity, as compared to age-matched Sprague-Dawley rats, mainly during the final stage of the experiment. Throughout the experiment, $\mathrm{P} 23 \mathrm{H}-1$ animals showed body temperature and locomotor activity circadian rhythms of decreased amplitude, weaker coupling strength, and higher rhythm fragmentation than that observed in wild-type animals. Conversely, body temperature and locomotor activity circadian rhythms were less robust in the elderly, as compared to young adult control Sprague-Dawley rats, which is in line with previous reports showing agedependent disturbances in the circadian system (Gubin et al., 2006; Tasaki et al., 2006).

Core-body temperature rhythms were more robust than circadian locomotor activity rhythms 
in both Sprague-Dawley and $\mathrm{P} 23 \mathrm{H}-1$ elderly rats.

These results are in agreement with previous studies showing that temperature rhythm is similar in juvenile, adult and senile mice, whereas the magnitude of the activity rhythm decreases as age increases (Weinert \& Waterhouse, 1999). The result is also in accordance with a previous study showing robust body temperature circadian rhythms in aged Sprague-Dawley and P23H-3 rats (Lax et al., 2011). In this sense, it has been postulated that both endogenous and environmental temperature cycles can participate in the synchronization of peripheral clocks in mammals (Brown et al., 2002).

Circadian impairments in $\mathrm{P} 23 \mathrm{H}-1$ rats correlated with a progressive deterioration of melanopsin-positive ganglion cell density and structure. Even though aged Sprague-Dawley rats (24 months of age) showed a mean density of mRGCs that was significantly lower than in younger animals, the mean density of mRGCs in $\mathrm{P} 23 \mathrm{H}-1$ rats at 18 and 24 months of age was significantly lower than that observed in agematched control Sprague-Dawley rats. Moreover, a progressive age-dependent deterioration of mRGC neurites was observed in $\mathrm{P} 23 \mathrm{H}-1$ rats. On the other hand, although mRGCs do not require synaptic inputs to generate light-induced signals, they receive these inputs from bipolar and amacrine cells (Belenky et al., 2003; Vugler et al., 2015). Therefore, the progressive loss of photoreceptors and the retinal remodeling in $\mathrm{P} 23 \mathrm{H}$ transgenic albino rats may also contribute to the deterioration of the animal's intrinsic response to light. All these results agree with previous findings in P23H-3 rats (Esquiva et al., 2013; Garcia-Ayuso et al., 2015) and represent further evidence of the existence of a positive correlation between the animals' visual capacity and the strength of their circadian rhythmicity.

Despite the alterations in the melanopsin system and circadian rhythms found in $\mathrm{P} 23 \mathrm{H}-1$ rats, all rhodopsin-mutant animals displayed 24-hentrained rhythms, probably supported by the considerable number of mRGCs survived, even in very advanced stages of retinal degeneration. Several authors have found data supporting the existence of an efficient survival system for mRGCs (Cui et al., 2015; Vugler et al., 2008). Both the molecular and cellular mechanism may be factors contributing to the resistance of mRGCs to cell injury, including the fact that they have a large soma, long and sparsely branching dendritic fields, intrinsic photosensitivity, pituitary adenylate cyclase-activating polypeptide (PACAP) expression, etc. (Cui et al., 2015; Li et al., 2008; Robinson \& Madison, 2004).

The present study demonstrates that degeneration of photoreceptors and inner retinal neurons have degenerative effects on the number and morphology of mRGCs and causes disturbances in the body temperature and locomotor activity circadian rhythms in $\mathrm{P} 23 \mathrm{H}-1$ rats. This leads us to conclude that vision loss in RP is correlated with progressive alterations in the melanopsin system and circadian rhythms. Future study is needed to determine the relationship between mRGC degeneration and impairment of circadian rhythms in RP patients.

\section{Funding}

This work is supported by the Spanish Ministry of Health [grant number FIS PI13-01124], the Spanish Ministry of Economy and Competitiveness [grant number MINECOFEDER BFU2012-36845], Instituto de Salud Carlos III [grant number RETICS-FEDER RD12/0034/0010], Organización Nacional de Ciegos Españoles (ONCE), and FUNDALUCE.

\section{Declaration of interest}

The authors report no conflicts of interest. The authors alone are responsible for the content and writing of the paper.

\section{References}

Belenky MA, Smeraski CA, Provencio I, Sollars PJ, Pickard GE. (2003). Melanopsin retinal ganglion cells receive bipolar and amacrine cell synapses. J Comp Neurol. 460:380-93.

Brown SA, Zumbrunn G, Fleury-Olela F, Preitner N, Schibler U. (2002). Rhythms of mammalian body temperature can sustain peripheral circadian clocks. Curr Biol. 12:1574-83.

Cuenca N, Fernandez-Sanchez L, Campello L, Maneu V, De la Villa P, Lax P, Pinilla I. (2014). Cellular responses following retinal injuries and therapeutic approaches for neurodegenerative diseases. Prog Retin Eye Res. 43:17-75.

Cuenca N, Kolb H. (1998). Circuitry and role of substance $\mathrm{P}$-immunoreactive neurons in the primate retina. J Comp Neurol. 393:439-56.

Cuenca N, Pinilla I, Sauve Y, Lu B, Wang S, Lund RD. (2004). Regressive and reactive changes in the connectivity 
patterns of rod and cone pathways of $\mathrm{P} 23 \mathrm{H}$ transgenic rat retina. Neuroscience. 127:301-17.

Cugini P, Cruciani F, De Rosa R, Pellegrino AM, Fontana S, Coda S, De Francesco GP, Mastromatteo A, Antonelli B, Vingolo EM, Regine F. (2001). Alterations of blood pressure and heart rate circadian rhythmic structure in nonblind patients affected by retinitis pigmentosa. J Hum Hypertens. 15:577-81.

Cui Q, Ren C, Sollars PJ, Pickard GE, So KF. (2015). The injury resistant ability of melanopsin-expressing intrinsically photosensitive retinal ganglion cells. Neuroscience. 284:845-53.

Dagnelie G. (2013). Age-related psychophysical changes and low vision. Invest Ophthalmol Vis Sci. 54:ORSF88-93.

de Zavalia N, Plano SA, Fernandez DC, Lanzani MF, Salido E, Belforte N, Sarmiento MI, Golombek DA, Rosenstein RE. (2011). Effect of experimental glaucoma on the nonimage forming visual system. J Neurochem. 117:904-14.

Dryja TP, McEvoy JA, McGee TL, Berson EL. (2000). Novel rhodopsin mutations Gly114Val and Gln184Pro in domi-

710 nant retinitis pigmentosa. Invest Ophthalmol Vis Sci. 41:3124-7.

Esquiva G, Lax P, Cuenca N. (2013). Impairment of intrinsically photosensitive retinal ganglion cells associated with late stages of retinal degeneration. Invest Ophthalmol Vis

715 Sci. 54:4605-18.

Fernandez DC, Sande PH, de Zavalia N, Belforte N, Dorfman D, Casiraghi LP, Golombek D, Rosenstein RE. (2013). Effect of experimental diabetic retinopathy on the nonimage-forming visual system. Chronobiol Int. 30:583-97.

720 Garcia-Ayuso D, Di Pierdomenico J, Esquiva G, NadalNicolas FM, Pinilla I, Cuenca N, Vidal-Sanz M, Agudo-Barriuso M, Villegas-Perez MP. (2015). Inherited photoreceptor degeneration causes the death of melanopsin-positive retinal ganglion cells and increases their coexpression of Brn3a. Invest Ophthalmol Vis Sci. 56:4592-604.

Garcia-Ayuso D, Salinas-Navarro M, Agudo M, Cuenca N, Pinilla I, Vidal-Sanz M, Villegas-Perez MP. (2010). Retinal ganglion cell numbers and delayed retinal ganglion cell death in the $\mathrm{P} 23 \mathrm{H}$ rat retina. Exp Eye Res. 91:800-10.

Gonzalez Fleitas MF, Bordone M, Rosenstein RE, Dorfman D. (2015). Effect of retinal ischemia on the non-image forming visual system. Chronobiol Int. 32:152-63.

Gooley JJ, Lu J, Chou TC, Scammell TE, Saper CB. (2001).

735 Melanopsin in cells of origin of the retinohypothalamic tract. Nat Neurosci. 4:1165.

Gordo MA, Recio J, Sanchez-Barcelo EJ. (2001). Decreased sleep quality in patients suffering from retinitis pigmentosa. J Sleep Res. 10:159-64.

740 Gubin DG, Gubin GD, Waterhouse J, Weinert D. (2006). The circadian body temperature rhythm in the elderly: effect of single daily melatonin dosing. Chronobiol Int. 23:639-58.

Hattar S, Liao HW, Takao M, Berson DM, Yau KW. (2002). Melanopsin-containing retinal ganglion cells: Architecture, projections, and intrinsic photosensitivity. Science.
Ionescu D, Driver HS, Heon E, Flanagan J, Shapiro CM. (2001). Sleep and daytime sleepiness in retinitis pigmentosa patients. J Sleep Res. 10:329-35.

Jean-Louis G, Zizi F, Lazzaro DR, Wolintz AH. (2008). Circadian rhythm dysfunction in glaucoma: A hypothesis. J Circadian Rhythms. 6:1.

Klein R, Klein BE. (2013). The prevalence of age-related eye diseases and visual impairment in aging: current estimates. Invest Ophthalmol Vis Sci. 54:ORSF5-ORSF13.

Kolomiets B, Dubus E, Simonutti M, Rosolen S, Sahel JA, Picaud S. (2010). Late histological and functional changes in the $\mathrm{P} 23 \mathrm{H}$ rat retina after photoreceptor loss. Neurobiol Dis. $38: 47-58$.

Lahouaoui H, Coutanson C, Cooper HM, Bennis M, DkhissiBenyahya O. (2014). Clock genes and behavioral responses to light are altered in a mouse model of diabetic retinopathy. PLoS One. 9:e101584.

Lax P, Esquiva G, Esteve-Rudd J, Otalora BB, Madrid JA, Cuenca N. (2012). Circadian dysfunction in a rotenoneinduced parkinsonian rodent model. Chronobiol Int. 29:147-56.

Lax P, Otalora BB, Esquiva G, Rol Mde L, Madrid JA, Cuenca N. (2011). Circadian dysfunction in $\mathrm{P} 23 \mathrm{H}$ rhodopsin transgenic rats: Effects of exogenous melatonin. J Pineal Res. 50:183-91.

Li SY, Yau SY, Chen BY, Tay DK, Lee VW, Pu ML, Chan HH, So KF. (2008). Enhanced survival of melanopsinexpressing retinal ganglion cells after injury is associated with the PI3 K/Akt pathway. Cell Mol Neurobiol. 28:1095-107.

Lockley SW, Arendt J, Skene DJ. (2007). Visual impairment and circadian rhythm disorders. Dialogues Clin Neurosci. 9:301-14.

Lucas RJ, Douglas RH, Foster RG. (2001). Characterization of an ocular photopigment capable of driving pupillary constriction in mice. Nat Neurosci. 4:621-6.

Machida S, Kondo M, Jamison JA, Khan NW, Kononen LT, Sugawara T, Bush RA, Sieving PA. (2000). P23H rhodopsin transgenic rat: correlation of retinal function with histopathology. Invest Ophthalmol Vis Sci. 41:3200-9.

Marc RE, Jones BW, Watt CB, Strettoi E. (2003). Neural remodeling in retinal degeneration. Prog Retin Eye Res. 22:607-55.

Mrosovsky N, Thompson S. (2008). Negative and positive masking responses to light in retinal degenerate slow (rds/rds) mice during aging. Vision Res. 48:1270-3.

Ortiz-Tudela E, Martinez-Nicolas A, Campos M, Rol MA, Madrid JA. (2010). A new integrated variable based on thermometry, actimetry and body position (TAP) to evaluate circadian system status in humans. PLoS Comput Biol. 6:e1000996.

Panda S, Provencio I, Tu DC, Pires SS, Rollag MD, Castrucci AM, Pletcher MT, Sato TK, Wiltshire T, Andahazy M, Kay SA, Van Gelder RN, Hogenesch JB. (2003). Melanopsin is required for non-image-forming photic responses in blind mice. Science. 301:525-7. 295:1065-70. 
Pinilla I, Fernández-Sánchez L, Segura FJ, Sánchez-Cano AI, M. TJ, L. F-B, Eellsh JT, Lax P, Cuenca N. (2015). Long time remodeling during retinal degeneration evaluated by Optical Coherence Tomography, immunocytochemistry and Fundus Autofluorescence Exp Eye Res (under revision).

Pinilla I, Lund RD, Sauve Y. (2005). Enhanced cone dysfunction in rats homozygous for the $\mathrm{P} 23 \mathrm{H}$ rhodopsin mutation. Neurosci Lett. 382:16-21.

Provencio I, Rodriguez IR, Jiang G, Hayes WP, Moreira EF, Rollag MD. (2000). A novel human opsin in the inner retina. J Neurosci. 20:600-5.

Puthussery T, Taylor WR. (2010). Functional changes in inner retinal neurons in animal models of photoreceptor degeneration. Adv Exp Med Biol. 664:525-32.

Quinn R. (2005). Comparing rat's to human's age: how old is my rat in people years? Nutrition. 21:775-7.

820 Robinson GA, Madison RD. (2004). Axotomized mouse retinal ganglion cells containing melanopsin show enhanced survival, but not enhanced axon regrowth into a peripheral nerve graft. Vision Res. 44:2667-74.

Skene DJ, Arendt J. (2007). Circadian rhythm sleep disorders in the blind and their treatment with melatonin. Sleep Med. 8:651-5.

Tasaki H, Serita T, Ueyama C, Kitano K, Seto S, Yano K. (2006). Long-Term follow-up of the circadian rhythm of heart rate and heart rate variability in healthy elderly patients. Circ J. 70:889-95.

Turek FW, Penev P, Zhang Y, van Reeth O, Zee P. (1995). Effects of age on the circadian system. Neurosci Biobehav Rev. 19:53-8.

Vugler A, Semo M, Ortin-Martinez A, Rojanasakul A, Nommiste B, Valiente-Soriano FJ, Garcia-Ayuso D, Coffey P, Vidal-Sanz M, Gias C. (2015). A role for the outer retina in development of the intrinsic pupillary light reflex in mice. Neuroscience. 286:60-78.

Vugler AA, Semo M, Joseph A, Jeffery G. (2008). Survival and remodeling of melanopsin cells during retinal dystrophy. Vis Neurosci. 25:125-38.

Weinert D, Waterhouse J. (1999). Daily activity and body temperature rhythms do not change simultaneously with age in laboratory mice. Physiol Behav. 66:605-12.

Witting W, Kwa IH, Eikelenboom P, Mirmiran M, Swaab DF. (1990). Alterations in the circadian rest-activity rhythm in aging and Alzheimer's disease. Biol Psychiatry. 27:563-72.

Zhang Y, Kornhauser JM, Zee PC, Mayo KE, Takahashi JS, Turek FW. (1996). Effects of aging on light-induced phaseshifting of circadian behavioral rhythms, fos expression and CREB phosphorylation in the hamster suprachiasmatic nucleus. Neuroscience. 70:951-61. 